\title{
Fitting Analysis of RGB Model of the Turquoise
}

\author{
Boling HUANG ${ }^{\mathrm{a}, \mathrm{b}, 1}$, Xuan WANG ${ }^{\mathrm{a}, \mathrm{b}}$, Tingting YANG ${ }^{\mathrm{a}, \mathrm{b}}$, Jiani SHEN ${ }^{\mathrm{a}, \mathrm{b}}$, Qingzheng \\ MA $^{\mathrm{a}, \mathrm{b}}$, Lansen LI ${ }^{\mathrm{a}, \mathrm{b}}$, Wei HUANG ${ }^{\mathrm{a}, \mathrm{b}}$, Miao LI ${ }^{\mathrm{a}, \mathrm{b}}$, Xihan YANG ${ }^{\mathrm{a}, \mathrm{b}}$ \\ ${ }^{a}$ Chongqing Academy of Metrology and Quality Inspection, Chongqing, China \\ ${ }^{\mathrm{b}}$ National Jewelry Testing Center (Chongqing), Chongqing, China
}

\begin{abstract}
In order to overcome the disadvantages of poor repeatability and strong subjectivity brought by traditional artificial experience classification and realize the quantitative intelligent classification of turquoise, RGB model needs to be fitted. By fitting the RGB model of turquoise classification with linear function and polynomial function, it is proved that after fitting the model with polynomial function, the Adjusted R-Square $\left(\mathrm{R}^{2}\right)$ reach more than 0.99. Using this method, the RGB model of optimal turquoise classification can be obtained, and improve the measurement accuracy of the color parameters of turquoise more than doubled.
\end{abstract}

Keywords. turquoise, RGB model, color parameters, machine vision system

\section{Introduction}

The turquoise is a basic phosphate mineral containing water, and it is a secondary mineral formed by the weathering and oxidizing-leaching of $\mathrm{Cu}$ and $\mathrm{P}$ minerals near the surface [1-2]. The chemical formula of turquoise is $\mathrm{CuAl}_{6}\left[\mathrm{PO}_{4}\right][\mathrm{OH}]_{8} \cdot 4 \mathrm{H}_{2} \mathrm{O}$. Turquoise is one of the oldest jade varieties, and the historical and archaeological studies at home and abroad have confirmed that turquoise has been widely used in religious, political and decorative fields since the neolithic age, and is widely popular in different cultural and social backgrounds [3-7]. In recent years, more and more consumers are pursuing highquality color and texture of turquoise as market demand continues to heat up, people's consumption level rises, as well as the needs of investment and collection. Turquoise is a typical colored jade whose color is the core element that classifing its quality, and the standardization of its color grading standard can provide beneficial support for regulating the market price of turquoise and promoting the healthy development of turquoise industry and craftsmanship [8].

Machine vision is one of the important technologies to realize digital color grading, including many disciplines such as image processing, optics and so on. As early as the 1980s, Sarkar [9] graded tomatoes based on color; Shearer [10] used machine vision to grade the color of sweet peppers; Liao [11] studied the color grading of corn kernels. There are relatively few studies on the color grading of jewelry and jade. Li [12] have studied the use of machine vision technology to grade the color of pearls.

1 Corresponding Author, Boling HUANG, Chongqing Academy of Metrology and Quality Inspection, No. 1 Yangliu North Road, Chongqing, China; Email: hbl_hot@126.com 
Based on a self-developed machine vision system for turquoise color grading, we found that the grading result of turquoise color has large deviations, and it is difficult to achieve accurate measurement. Thus, after fitting analysis of the RGB model for measuring turquoise color, the most suitable model was calculated. Using this model, accurate and stable turquoise color parameters can be obtained, and accurate digital measurement of turquoise color can be achieved.

\section{Principle of Curve Fitting Function}

\section{1. linear function}

Linear function is the basic method of data processing, and it is also a method to solve the problem that the number of equations is more than the number of variables when fitting the regression line. The fitting function is as follows:

$$
\mathrm{y}_{(R G B)}(x)=a x_{(R G B)}+b
$$

$y_{(R G B)}(x)$ is the fitting result of the R, G, B; $x_{(R G B)}$ is the measured value; the $\mathrm{a}$ and $\mathrm{b}$ are constants.

\section{2. polynomial function}

In order to make the fitting curve closer to the actual color, n-order polynomial is used to fit the various color parameters extracted in the actual situation. The fitting function is as follows:

$$
f_{(R G B)}(x)=\mathrm{a}_{0}+\mathrm{a}_{1} x_{(R G B)}+\cdots+\mathrm{a}_{n} x_{(R G B)}^{n}
$$

$f_{(R G B)}(x)$ is the fitting result of the $\mathrm{R}, \mathrm{G}, \mathrm{B} ; x_{(R G B)}$ is the measured value; $\mathrm{n}$ is the number of times; $\mathrm{a}_{n}$ is a constant .

For the degree $\mathrm{n}$, it can be obtained from the polynomial property that the $\mathrm{n}$ selection is higher, the function fitting effect is better, the error is smaller, but too high will lead to more and more serious overfitting. In order to prevent overfitting and ensure small error, the same finite number was used to fit R, G, B color parameters.

\section{Analysis of R, G, B Color Parameters}

\subsection{Experiment Preparation}

The color parameters of the sample are extracted by using the machine vision system developed by the research team of this paper based on the color grading of turquoise. Due to the influence of uncertain factors such as environment and operation, the color parameters of the same sample at different times and different personnel are greatly changed, which cannot meet the accurate and stable determination of turquoise color. In order to ensure the accuracy and stability of the data, the standard color plate was used to calibrate and fit the RGB color model of turquoise. The color parameters of the standard color plate are shown in table 1 . The color parameters in the standard color plate 
were measured. Each color plate was measured five times in a relatively short time (within $3 \mathrm{~min}$ ).

Table 1. The color parameters of standard color plate

\begin{tabular}{cccc}
\hline & \multicolumn{3}{c}{ color parameters } \\
\cline { 2 - 4 } No. & R & G & B \\
\hline Whe & 243 & 243 & 242 \\
Nel 1 & 200 & 200 & 200 \\
Nel 2 & 160 & 160 & 160 \\
Nel 3 & 122 & 122 & 121 \\
Nel 4 & 85 & 85 & 85 \\
Blk & 52 & 52 & 52 \\
\hline
\end{tabular}

\subsection{Linear Function Fitting}

The linear function is used to fit the measured color parameters of the standard color plate, and the results are shown in table 2.

Table 2. Linear function fitting results

\begin{tabular}{cccc}
\hline & \multicolumn{3}{c}{ color parameters } \\
\cline { 2 - 4 } & $\mathbf{R}$ & $\mathbf{G}$ & $\mathbf{B}$ \\
\hline $\mathrm{a}$ & 1.29 & 1.30 & 1.30 \\
$\mathrm{~b}$ & -75.79 & -74.92 & -74.32 \\
RSS & 7302 & 6306 & 6158 \\
Pearson's r & 0.9832 & 0.9857 & 0.9859 \\
Adjusted R-Square & 0.9656 & 0.9706 & 0.9711 \\
\hline
\end{tabular}

RSS is the residual sum of squares, which is the amount of calculation to measure the fitting degree of the linear function model. It can be seen from the table that the RSS values of color parameters R, G and B are relatively large, indicating that the fitting degree of the function is not high.

Pearson 's $\mathrm{r}$ is the correlation coefficient and Adjusted R-Square is the degree of freedom determination coefficient. The value is the amount of calculation to measure the linear relationship between variables and the explanatory power of variable $\mathrm{x}$ in the fitting function to the original function $y$. The closer the value is to 1 , the better the fitting effect of the model is, the more accurate the data is. It can be seen from the table that the linear function has a certain fitting effect on the color parameters $\mathrm{R}, \mathrm{G}$ and $\mathrm{B}$.

Based on the above data, it can be seen that although the linear function is the basic method of data processing, the fitting degree of the RGB color model of turquoise is not good, and the accurate and stable color parameters of R, G and B cannot be obtained by this function. Therefore, the linear function is not suitable for this model. 


\subsection{Polynomial Function}

The polynomial function is used to fit the measured color parameters of the standard color plate. For the n, the polynomial property can be obtained. The higher the $\mathrm{n}$ selection is, the better the function fitting effect is, and the smaller the error is. However, too high will lead to more and more serious overfitting. Table 3 to table 5 is the result of polynomial fitting with different degrees.

Table 3. Polynomial function fitting results of color parameters of $\mathrm{R}$

\begin{tabular}{ccccc}
\hline \multicolumn{5}{c}{$\mathbf{R}$} \\
\hline $\mathrm{n}$ & 2 & 3 & 4 & 5 \\
$\mathrm{a}_{0}$ & -3.14 & 33.53 & 27.17 & 146.04 \\
$\mathrm{a}_{1}$ & 0.057 & -0.938 & -0.703 & 7.289 \\
$\mathrm{a}_{2}$ & 0.004 & 0.012 & 0.009 & -0.126 \\
$\mathrm{a}_{3}$ & - & -0.00002 & -0.000003 & 0.00105 \\
$\mathrm{a}_{4}$ & - & - & -0.00000002 & -0.000004 \\
$\mathrm{a}_{5}$ & - & - & - & 0.000000005 \\
RSS & 360 & 68 & 67 & 16 \\
Adjusted R-Square & 0.9982 & 0.9997 & 0.9997 & 0.9999 \\
\hline
\end{tabular}

Table 4. Polynomial function fitting results of color parameters of $\mathrm{G}$

\begin{tabular}{ccccc}
\hline \multicolumn{5}{c}{ G } \\
\hline $\mathrm{n}$ & 2 & 3 & 4 & 5 \\
$\mathrm{a}_{0}$ & -9.68 & 42.718 & 11.371 & -187.635 \\
$\mathrm{a}_{1}$ & 0.193 & -1.230 & -0.073 & 9.110 \\
$\mathrm{a}_{2}$ & 0.004 & 0.145 & 0.0005 & -0.154 \\
$\mathrm{a}_{3}$ & - & -0.00002 & 0.00005 & 0.00125 \\
$\mathrm{a}_{4}$ & - & - & -0.0000001 & -0.000005 \\
$\mathrm{a}_{5}$ & - & - & - & 0.000000006 \\
RSS & 709 & 113 & 87 & 19 \\
Adjusted R-Square & 0.9966 & 0.9994 & 0.9995 & 0.9999 \\
\hline
\end{tabular}

Table 5. Polynomial function fitting results of color parameters of B

\begin{tabular}{ccccc}
\hline \multicolumn{5}{c}{ B } \\
\hline $\mathrm{n}$ & 2 & 3 & 4 & 5 \\
$\mathrm{a}_{0}$ & -8.349 & 38.462 & 8.988 & -103.703 \\
$\mathrm{a}_{1}$ & 0.177 & -1.096 & -0.006 & 5.193 \\
$\mathrm{a}_{2}$ & 0.004 & 0.014 & 0.0002 & -0.087 \\
$\mathrm{a}_{3}$ & - & -0.00002 & 0.00004 & 0.0007 \\
$\mathrm{a}_{4}$ & - & - & -0.0000001 & -0.000003 \\
$\mathrm{a}_{5}$ & - & - & - & 0.000000003 \\
RSS & 532 & 58 & 36 & 14 \\
Adjusted R-Square & 0.9974 & 0.9997 & 0.9998 & 0.9999 \\
\hline
\end{tabular}

According to the analysis in the above table, the polynomial function has a high fitting effect on color parameters, and the fitting effect is high when $n \geq 2$. However, when $\mathrm{n}>2$, the coefficient value of each number of times is too small, and the improvement of fitting effect is very small. Therefore, when fitting the function, selecting $\mathrm{n}=2$ for function fitting can not only effectively ensure the matching degree of color parameters, but also select $\mathrm{n}=2$ for fitting, which can better adapt the fitting function to different colors and avoid over fitting.

\subsection{Rgb Model of the Turquoise}

The parameter distribution and fitting results of fitting polynomial function models of $\mathrm{R}$, $\mathrm{G}$ and $\mathrm{B}$ can be obtained by polynomial function $(\mathrm{n}=2)$, as shown in table 6 . 
Table 6. The fitting parameter distribution and results of $R, G, B$

\begin{tabular}{cccccc}
\hline $\begin{array}{c}\text { color } \\
\text { parameters }\end{array}$ & $\mathbf{a}$ & a1 & a2 & RSS & $\begin{array}{c}\text { Adjusted R- } \\
\text { Square }\end{array}$ \\
\hline R & -3.14 & 0.057 & 0.004 & 360 & 0.9982 \\
G & -9.68 & 0.193 & 0.004 & 709 & 0.9966 \\
B & -8.349 & 0.177 & 0.004 & 532 & 0.9974 \\
\hline
\end{tabular}

It can be seen from table 5 that the degree of freedom determination coefficient is close to the limit value 1 . It is considered that the fitting method of turquoise RGB color model using quadratic polynomial is feasible, and the obtained color parameters are accurate and stable.

\section{Confirmation Experiment}

In order to verify the feasibility of fitting the RGB color model of turquoise with quadratic polynomial function, appropriate turquoise should be selected to test its color parameters. However, since there is no turquoise standard sample to calibrate the color parameters at present, we select the standard color card closest to the color of turquoise according to the national standard for testing [13]. The R, G, and B of the standard color card are 114, 178, and 212, respectively. The test instrument is carried out with the selfdeveloped machine vision system for turquoise color grading, and the test number is 11 . The results are shown in table 7 .

Table 7. The fitting parameter distribution and results of R, G, B

\begin{tabular}{ccccccc}
\hline & \multicolumn{2}{c}{$\mathbf{R}$} & \multicolumn{2}{c}{$\mathbf{G}$} & \multicolumn{2}{c}{ B } \\
\cline { 2 - 6 } & $\begin{array}{c}\text { measured } \\
\text { value }\end{array}$ & $\begin{array}{c}\text { fitting } \\
\text { values }\end{array}$ & $\begin{array}{c}\text { measured } \\
\text { value }\end{array}$ & $\begin{array}{c}\text { fitting } \\
\text { values }\end{array}$ & $\begin{array}{c}\text { measured } \\
\text { value }\end{array}$ & $\begin{array}{c}\text { fitting } \\
\text { values }\end{array}$ \\
1 & 82 & 136 & 134 & 171 & 166 & 191 \\
2 & 88 & 142 & 130 & 168 & 161 & 188 \\
3 & 87 & 140 & 126 & 165 & 159 & 187 \\
4 & 90 & 142 & 130 & 168 & 163 & 189 \\
5 & 94 & 146 & 134 & 171 & 165 & 190 \\
6 & 89 & 142 & 128 & 167 & 159 & 187 \\
7 & 87 & 140 & 127 & 166 & 159 & 187 \\
8 & 88 & 141 & 126 & 165 & 158 & 186 \\
9 & 90 & 142 & 134 & 171 & 165 & 190 \\
10 & 85 & 142 & 130 & 168 & 162 & 188 \\
11 & 87 & 140 & 126 & 165 & 158 & 186 \\
RE & $23 \%$ & $23 \%$ & $27 \%$ & $6 \%$ & $24 \%$ & $11 \%$ \\
SD & 3.0 & 3.3 & 3.0 & 2.5 & 2.2 & 1.8 \\
Ave & 88 & 161 & 130 & 168 & 161 & 188 \\
RSD & $3.5 \%$ & $2.5 \%$ & $1.9 \%$ & $1.8 \%$ & $1.3 \%$ & $1.0 \%$ \\
\hline
\end{tabular}

According to the analysis in the above table, by fitting the color parameters with quadratic polynomial function, in addition to the $\mathrm{R}$ color parameter, the accuracy of $\mathrm{G}$ and $\mathrm{B}$ parameters is more than doubled, and the precision is doubled. Therefore, the optimal model calculated by using quadratic polynomial function fitting analysis can obtain accurate and stable turquoise color parameters, and realize accurate digital classification of turquoise color. 


\section{Conclusion}

In order to improve the accurate and stable measurement of turquoise color parameters by machine vision system, the quadratic polynomial function is used to fit and analyse the RGB model of turquoise color measurement, and the most suitable model is calculated. The experimental results show that the accurate and stable turquoise color parameters can be obtained by using the model, which can improve the accuracy and precision of measuring turquoise color parameters more than twice, and realize the digital measurement of turquoise color.

\section{Acknowledgments}

Thanks to the scientific research plan of Chongqing Administration for Market Regulation (CQSJKJ2020001; CQSJKJ2021023) for supporting this research.

\section{References}

[1] She Lingzhu, Qin Ying, Feng Min, et al. Spectroscopy and Spectral Analysis, 2008(09):157-160.

[2] Li Jianjun, Li Guihua, Fan Chengxing, et al. Superhard Material Engineering, 2016, 28(02):61-65.

[3] Ye Xiaohong, Ren Jia, Xu Hong, et al. Quaternary Sciences, 2014, 034(001):212-223.

[4] Li Danxiang, Xian Yiheng. Sciences of conservation and archaeology, 2015(02):104-111.

[5] Hao Yongwei, Hao Feizhou. Acta Petrologica et Mineralogica, 2002, 21(S):147-150.

[6] Chen Q, Yin Z, Qi L, et al. Turquoise from Zhushan County, HubeiProvince, China. Gems \& Gemology, 2012, 48(3):198-204.

[7] Fuquan W. A Gemological Study of Turquoise in China. Gems and Gemology, 1986, 22(1):35-37.

[8] Luo Zemin, Shen Xitian, Zhu Qinwen, et al. Journal of Gems \& Gemmology, 2016, 018(002):1-8.

[9] Rigney M P , Kranzler G A . Machine Vision for Grading Southern Pine Seedlings. Transactions of the Asae, 1991, 31(2):0642-46.

[10] Shearer S A, Payne F A. Transactions of the ASAE, 1990, 33(6):1245-50.

[11] Liao, K, Li, Z, Reid, J.F, et al. Knowledge-based color discrimination of corn kernels. Paper American Society of Agricultural Engineers, 1992.

[12] Li Yueqiao, Tu Wangping, Shi Yang, et al. China Plant Engineering, 2019(18):113-114.

[13] GB/T 36169-2018 Turquoise-Grading. Beijing: National Standard Press, 2018. 